\title{
Flow effects on the loop bridge ratio in sphere-forming triblock copolymer
}

\author{
Yoshiaki Takahashi ${ }^{1}$ and Satoshi Hasegawa ${ }^{2}$
}

\section{Institute for Materials Chemistry and Engineering, Kyushu University, Fukuoka, Japan \\ 2. Department of Molecular \& Materials Sciences, Kyushu University, Fukuoka, Japan}

\begin{abstract}
Anisotropy of the bridge fraction of middle isoprene block chains in sheared films of sphere-forming polystyrene- $b$-polyisoprene- $b$-polystyrene triblock copolymers in different directions are examined by breaking stress under simple elongation measurements at a fast draw rate, which reflect the bridge fraction. There was no practical difference between the data obtained for the films cut in the directions parallel and perpendicular to the shear flow direction at each shear rate. The breaking stress for the samples sheared at high shear rates become slightly lower than those obtained at low sher rates. The absolute values and the difference is consistent with the previous study, in which pull out of end blocks due to the flow is examined. Thus, we conclude that when flow is applied to sphere-forming triblocks, only small portion of end blocks are pulled out to flow and there is no orientation dependent anisotropy of bridge fractions.
\end{abstract}

Key words: Poly(styrene-block-isoprene-block-styrene), Sphere-forming triblock copolymer, Thermoplastic elastomer, Bridge Fraction, Flow Effects

\section{INTRODUCTION}

In general, there exist bridge, loop and dangling chains in polymer networks such as gels and rubbers. Those sub-chains may differently affect the mechanical properties of the material. However, there was no systematic study of this problem since the ratio of these chains cannot be determined.

Sphere-forming triblock copolymers with glassy or crystalline end blocks and rubbery middle block, known as thermoplastic elastomers are suitable model systems to study the above problem. Living anionic polymerization technique enabled us to prepare special middle block chains, which can be used to determine and/or control the bridge, loop, and dangling end fractions as mentioned below.

A pioneering study was performed by Watanabe et al. [1-4]. Polystyrene- $b$-polyisoprene- $b$-polystyrene (SIS) tribrock copolymers with dipole-inverted polyisoprene chains are prepared by coupling of living SI diblocks. They determined the bridge fraction of I chains for lamellar-forming SIS and sphere-forming SIS triblock copolymers by dielectric relaxation measurements.

Takano et al. [5,6] prepared a series of samples having almost the same size of spherical microdomains but different loop/bridge fractions by mixing cyclic-SI (C-SI) diblock copolymers and SIS, which is the precursor of C-SI. They also prepared a $100 \%$ bridged reference, i.e., polystyrene- $b$-polyisoprene- $b$-poly(2-vinylpiridine) (SIP) triblock copolymer and determined the bridge fraction of the I chains in SIS and SIS/C-SI blends from Young's moduli obtained by dynamic elongational measurements assuming linear proportionality of loop chain fraction to the C-SI content. The bridge fraction thus determined was confirmed by breaking stress under fast draw elongations. Furthermore, another series of samples with different bridge/dangling chain fractions are prepared by mixing SIP and equal amounts of SI and IP diblocks and the effects of dangling chains on Young's modulus are examined by almost the same methods mentioned above [7]. It was reported that there is no practical difference between the roles of loop and dangling chains.

Shear and annealing effects on the elastic behavior for the same SIS and SIP are also examined by dynamic mechanical analysis [8]. Plateau modulus $G_{\mathrm{N}}$ obtained at $70{ }^{\circ} \mathrm{C}$ rapidly increased within the first one hour, while $G_{\mathrm{N}}$ very gradually increased at longer time. By comparing the data with and without shear, the initial increase of $G_{\mathrm{N}}$ is attributed to increase of restoring force of the lattice caused by the relaxation of lattice distortion. The gradual increase at longer time is attributed to reformation of network structure by reentrant of pulled-out end blocks into the spherical domains since it was faster for SIS than SIP. It was concluded that the effects of pull out of end blocks and network reformation on elastic properties for molded film are not so large.

The above study was carried out neglecting the possibility of anisotropic alignments of molecular backbones, which might be introduced during the flow. Flow-induced alignments of block copolymer chains have been studied especially for lamellar-forming diblock copolymers [9]. It is reported that those chains flow like dumbbells rolling around the backbones to maintain microphase separated structure. Therefore, thereis a possibility that the sphere-forming triblocks also have orientation dependent bridge fraction after the flow.

In this study, we examine the anisotropy of orientation of chain backbones in sheared SIS block copolymer films. Stress-strain curves are obtained for sheared films deformed under different shear rate but equal strain are cut in two different directions and examined. 


\section{EXPERIMENT}

\subsection{Sample preparation}

Triblock copolymer sample used in this study is the same SIS used in the previous studies [1,2]. The details of preparation and characterization of the samples are already reported [1]. The number-averaged molecular weight, $M_{\mathrm{n}}$, molecular weight distribution index, $M_{\mathrm{w}} / M_{\mathrm{n}}$, where $M_{\mathrm{w}}$ is weight-averaged molecular weight, and volume fraction of isoprene, $\phi_{\mathrm{I}}$ are $2.0 \times 10^{5}, .1 .03$, and 0.9 , respectively. It was confirmed by TEM observation that this block copolymer posses isolated spherical domains of polystyrene end blocks.

\subsection{Measurements}

Sample films are prepared by hot-press at $150{ }^{\circ} \mathrm{C}$ for 5 minutes. The pressed films are cut into a rectangular shape $(1 \mathrm{~mm} \times 10 \mathrm{~mm} \times 10 \mathrm{~mm})$ and sheared at different shear rates $\left(5-15 \mathrm{~s}^{-1}\right)$ by a home made shearing device, keeping the total strain at 2.5. Then the sheared films are cut to test films with size of $1 \mathrm{~mm} \times 7 \mathrm{~mm} \times$ $150 \mu$ in two different directions as shown in Figure 1. That is, the rectangular surface areas of the films are parallel and perpendicular to the flow direction. Hereafter, we simply call these films as parallel and perpendicular films, respectively. Breaking stress of the test films under simple elongation are measured with a Rheometrics Minimat 2000 at room temperature. The drawing rate was $90 \mathrm{~cm} / \mathrm{min}$. The breaking stress measurements are performed for several times for each sample.
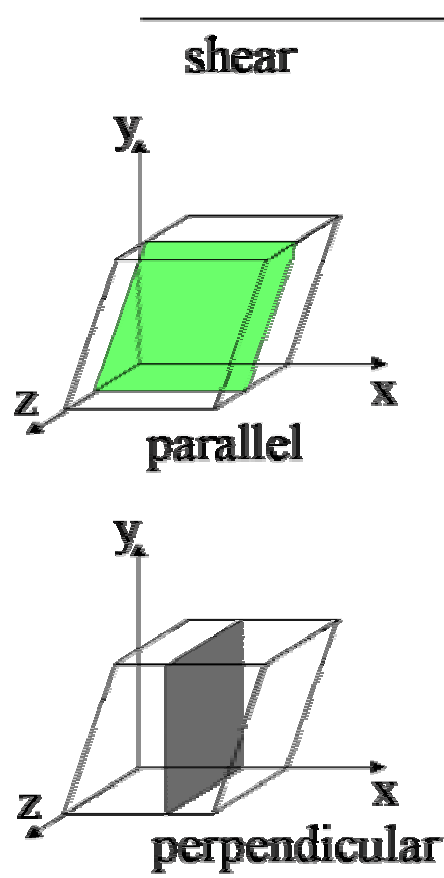

Figure 1. Cutting direction of films.

\section{RESULTS AND DISCUSSIONS}

Figure 2 shows examples of stress strain curves (S-S curve) for the films prepared at the same condition. There is a data which is much different from others. This kind of different behavior is observed when microcrack generates during elongation, as pointed out in the previous pa- per [6]. We chose the reliable data by using reduced S-S curve, in which stress and strain are reduced by breaking point values. When there is no or only little effect of microcrack generation, S-S curve should compose a single line since the deformation process must be the same. The data which did not compose universal line are eliminated from the following analysis and discussion.

Figure 3 shows examples of S-S curves thus selected. Roughly speaking, there is no obvious difference among these S-S curves. To compare the data more systematically, averaged S-S curves are obtained for parallel and perpendicular directions at each shear rate.

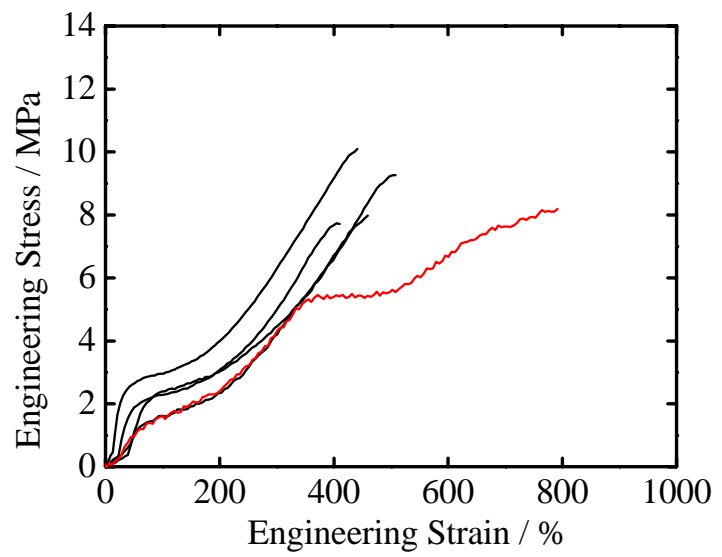

Figure 2. Examples of stress-strain curves for samples prepared at a same condition $\left(10 \mathrm{~s}^{-1}\right)$.
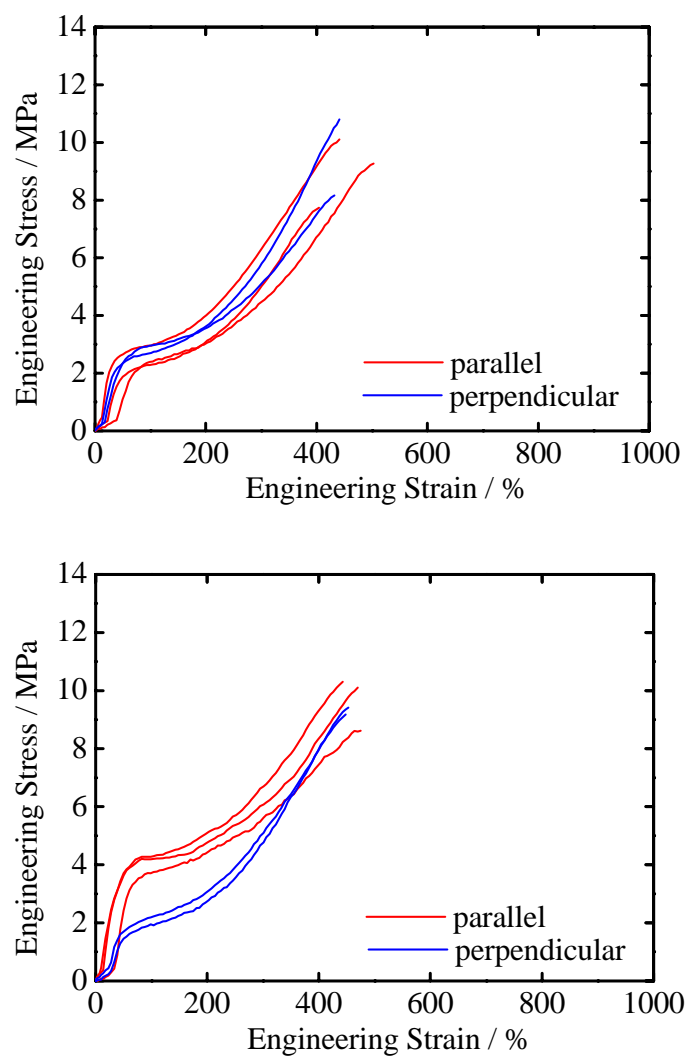

Figure 3. Stress-strain curves without the effects of microcrack generation. Shear rates are ::10 $\mathrm{s}^{-1}$ (top) and 15 $\mathrm{s}^{-1}$ (bottom). 
Figure 4 shows averaged S-S curves in the each direction at the different shear rates. It is clear that all S-S curves except that sheared at the highest shear rate (15 $\mathrm{s}^{-1}$ ) well coincide with each other irrespective of shear rates and directions. This result implies that the sheared films prepared at low shear rates are uniform. The S-S curve obtained for $15 \mathrm{~s}^{-1}$ in the parallel direction is slightly higher than others at low strain (say $<300 \%$ ). The difference can be attributed to the regularity of the spherical domain lattice. That is, when the regularity of the lattice is fine, the restoring force against deformation of lattice become strong which arises at strain lower than $300 \%$ [2]. Moreover, it is reported that the shear flow aligns the lattice [10]. We confirmed by SAXS measurements that the regularity of lattice for the above film become slightly fine (higher order peak become clear).
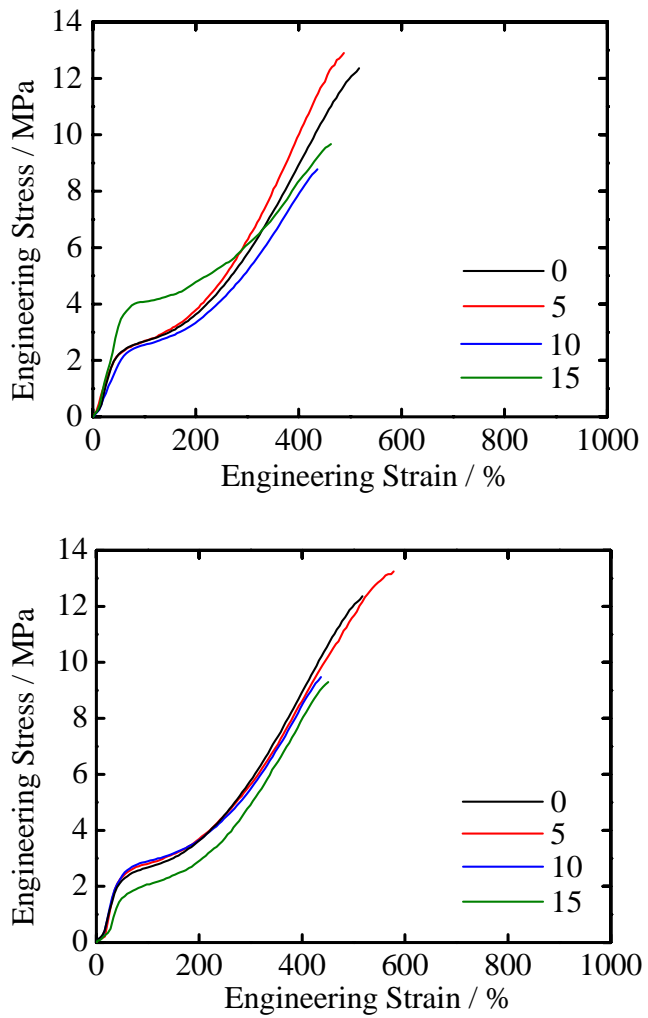

Figure 4. Averaged S-S curves for (top) parallel and (bottom) perpendicular films at different shear rates.

To discuss the fractions of bridged middle chains and their anistropy in the sheared films, average values of breaking stress for parallel and perpendicular directions at each shear rate are obtained. Figure 5 shows plots of the averaged breaking stress vs. shear rate for all the samples. There is no practical difference in the parallel and perpendicular directions at each shear rate. By comparing the data at different shear rates, it can be pointed out that the values at higher shear rates are slightly lower than those for lower shear rates. Corresponding breaking strains are also slightly lower, though the features of S-S curves are almost the same (see Fig. 3). In a previous paper [8], we discussed the flow and annealing effects on the elasticity of SIS and SIP. We reported that only small amount of end blocks are needed to be pulled out to flow, maintaining the microphase separated structure at melt states.
Once the smooth flow become possible, there is no need to pull out more end blocks for successive flow. The values in Fig. 5 are consistent with the values reported in that study. Therefore, slightly lower values at high shear rates can be attributed to the pulled out end blocks; when $\mathrm{S}$ end block is pulled out from $\mathrm{S}$ domain and become either dangling chains or loop chains, the connected I chains does not work as bridged chains any more.

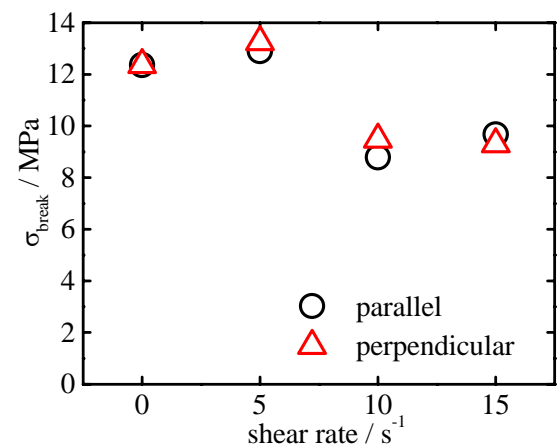

Figure 5. Plots of breaking stress vs. shear rates.

\section{CONCLUSIONS}

Anisotropy in the bridged chain fractions for sheared SIS in the different directions are examined by breaking stress measurements. There was no practical difference between the data obtained for the films cut in the directions parallel and perpendicular to the shear flow direction at each shear rate. The breaking stress for the samples sheared at high shear rates become slightly lower than those obtained at low sher rates. The absolute values and the difference is consistent with the previous study, in which pull out of end blocks due to the flow is examined. Thus, we conclude that when flow is applied to sphere-forming triblocks, only small portion of end blocks are pulled out to flow and there is no orientation dependent anisotropy of bridge fractions.

\section{REFERENCES}

1. H. Watanabe, Macromolecules, 28 (1995), p.5006.

2. H. Watanabe, T. Sato, K. Osaki, M-L. Yao, A. Yamagishi, Macromolecules, 30 (1997), p.5877.

3. H. Watanabe, T. Sato, K. Osaki, Y. Matsumiya, S. H. Anastasiadis, Nihon Reoroji Gakkaishi (J. Soc. Rheol. Jpn), 27 (1999), p.173.

4. H. Watanabe, T. Sato, K. Osaki, Macromolecules, 33 (2000), p.2545.

5. Takano, I. Kamaya, Y. Takahashi, Y. Matsushita, Macromolecules, 38 (2005), p.9718.

6. Y. Takahashi, Y. Song, N. Nemoto, A. Takano, Y. Akazawa, Y. Matsushita, Macromolecules, 38 (2005), p. 9724.

7. Y. Takahashi, Y. Akazawa, A. Takano, and Y. Matsushita, Polym. J. 36, (2006) p. 603.

8. Y. Takahashi, S. Yahata, A. Takano, and Y. Matsushita, Nihon Reoroji Gakkaishi (J. Soc. Rheol. Japan), 34 (2006), p.177.

9. Y. Takahashi, M. Naruse, Y. Akazawa, A. Takano, and Y. Matsushita, Polym. J., 37 (2005), p.900.

10. C. Daniel, I. W. Hamely, and K. Mortensen, Polymer, 41, (2000) p.9239. 